\title{
ASO Perspectives: Adjuvant Nivolumab in Resected Esophageal or Gastroesophageal Junction Cancer: Never Stop Questioning
}

\author{
Bryan J. Schneider, $\mathrm{MD}^{1}$, and Andrew C. Chang, $\mathrm{MD}^{2}$ \\ ${ }^{1}$ Department of Internal Medicine, University of Michigan, Ann Arbor, MI; ${ }^{2}$ Department of Surgery, University of \\ Michigan, Ann Arbor, MI
}

\begin{abstract}
This ASO perspective reviews the findings of a randomized placebo-controlled, clinical trial evaluating adjuvant nivolumab in esophageal or gastroesophageal junction carcinoma, reported recently by the Checkmate 577 investigators. The use of postoperative immunotherapy represents a significant paradigm shift for managing patients who have had limited evidence-based treatment options after completing neoadjuvant chemoradiation followed by resection for these aggressive malignancies.
\end{abstract}

Despite major advances in the evaluation and management of esophageal or gastroesophageal junction carcinoma, overall 5-year survival following treatment for this aggressive malignancy remains poor: $20 \%$ for all stages, $26 \%$ for patients diagnosed with regional disease, and $47 \%$ for those with localized disease. ${ }^{1}$ High-level evidence supports the use of multimodality therapy for patients with resectable, locoregionally advanced, AJCC/ UICC stage 2 or 3, esophageal or gastroesophageal junction cancers. ${ }^{2,3}$ The ChemoRadiotherapy for Oesophageal cancer followed by Surgery Study (CROSS) group has reported that patients with resectable, locally advanced esophageal or esophagogastric junction carcinoma who receive neoadjuvant weekly carboplatin, paclitaxel, and concurrent radiation therapy followed by esophagogastrectomy have improved median overall survival of 48.6 months compared with 24.0 months for those undergoing

(C) Society of Surgical Oncology 2022

First Received: 4 August 2021

Accepted: 14 December 2021;

Published Online: 19 February 2022

A. C. Chang, MD

e-mail: andrwchg@umich.edu surgery only (hazard ratio [HR] $0.68 ; 95 \%$ confidence interval $[\mathrm{CI}]$ 0.53-0.88; log-rank $p=0.003) .^{2}$ Alternatively, perioperative chemotherapy given before and after resection is an acceptable standard of care as demonstrated by the landmark trials reported by Cunningham et al. ${ }^{4}$ and Ychou et al..$^{5}$ Recently, the FLOT4-Arbeitsgemeinschaft Internistische Onkologie (AIO) trial demonstrated improved clinical benefit with oxaliplatin, fluorouracil, and docetaxel before and after resection with a median overall survival of 50 months versus 35 months with perioperative epirubicin, cisplatin, and fluorouracil (IV or oral) (HR 0.77; 95\% CI $0.63-0.94){ }^{3}$

Unfortunately, the majority of patients with locally advanced, resectable disease remain at risk for recurrent malignancy that is almost uniformly incurable. The CROSS group observed pathologic complete response (pCR) in $29 \%$ of subjects (47 of 161) who had received neoadjuvant chemoradiation followed by resection, with a greater rate $(p=0.008)$ of $\mathrm{pCR}$ reported for patients with squamous cell histology $(49 \% ; 18$ of 37$)$ versus patients with adenocarcinoma (23\%; 28 of 121). ${ }^{6}$ A subsequent report of long-term follow-up demonstrated a median overall survival of 48.6 months in the neoadjuvant chemoradiotherapy-plus-surgery group and 24.0 months in the surgery-alone group (HR $0.68 ; 95 \%$ CI $0.53-0.88$, logrank $p=0.033){ }^{2}$ Median progression-free survival was 37.7 months in the neoadjuvant chemoradiotherapy-plussurgery group and 16.2 months in the surgery-alone group (HR 0.64; 95\% CI 0.49-0.82; log-rank $p=0.000217$ ). Locoregional and/or distant disease recurrence was reduced with neoadjuvant treatment; however, 87 of 178 subjects $(49 \%)$ still progressed despite trimodality therapy versus 124 of 188 subjects $(66 \%)$ with resection alone (HR $0.58 ; 95 \%$ CI $0.44-0.76, p<0.0001)$. 
Improved histopathologic response also was reported by the FLOT4-AIO investigators in patients receiving FLOT versus ECF/ECX chemotherapy, despite similar pretreatment clinical $\mathrm{T}$ and $\mathrm{N}$ stage distributions between the treatment arms. Trials evaluating perioperative chemotherapy, however, have included patients with gastric and gastroesophageal junction adenocarcinoma and might not be translatable to patients with adenocarcinoma of the esophagus or squamous cell carcinoma of the esophagus/gastroesophageal junction. ${ }^{3-5}$

Retrospective studies suggest adjuvant systemic therapy may improve survival for patients at high risk of recurrence after induction therapy and complete (R0) resection, particularly for the majority of patients who do not achieve a pathologic complete response. ${ }^{7,8}$ However, these retrospective studies are difficult to interpret given the limited data that describes the neoadjuvant treatments previously received, why adjuvant treatment was recommended, and what adjuvant chemotherapy regimens were administered. For example, Samson et al. ${ }^{7}$ identified 3,100 patients with pathologic positive lymph nodes after undergoing induction therapy followed by esophagectomy over the period from 2006 to 2012. Of these patients, 475 (15.3\%) received adjuvant chemotherapy. Factors associated with receiving adjuvant therapy included adenocarcinoma histology and increasing pathologic node status, whereas patients who had received neoadjuvant chemoradiation (compared with chemotherapy alone), increasing age, and longer postresection hospital lengths of stay were less likely to have received subsequent treatment. In this national cohort, adjuvant chemotherapy was associated with improved median overall survival of 30.8 months, compared with 23.0 months for those who had not received postoperative treatment (HR 0.71; 95\% CI 0.59-0.87, $p<0.001$ ). To adjust for potential selection and allocation bias, propensity scoring, including factors, such as patient demographic data, comorbidity index, hospital length of stay, readmission status, as well as surrogates for social determinants of health, was completed. In a subset of 1,445 patients who had complete data for propensity score matching, a similar proportion, $215(14.8 \%)$, received adjuvant therapy. In 1:1 propensity score-matched cohorts, median overall survival remained significantly greater for patients who had received adjuvant therapy compared with those matched patients who had not (33.1 vs. 26.2 months; $p<0.03$ ).

In a retrospective study that included 9 collaborating centers, Semenkovich et al. ${ }^{8}$ identified 1,082 patients with residual lymph node involvement who had received induction therapy followed by esophagectomy. In this cohort, 940 (87\%) had received preoperative chemoradiation and 140 (13\%) had received chemotherapy. Adjuvant therapy was administered in 209 (19.3\%) patients, consisting predominantly of chemotherapy alone in 167
(79.9\%), chemoradiotherapy in $30(14.4 \%)$, and radiation only in $12(5.7 \%)$ patients. When comparing treatment strategies with their induction therapy, adjuvant chemotherapy regimens were similar in $39(19 \%)$, different in $99(47 \%)$, and undetermined in $71(34 \%)$ patients. Patients receiving adjuvant therapy had longer median overall survival of 2.6 years vs. 2.3 years (Wilcoxon $p=$ 0.02 ; $\log$-rank $p=0.06$ ). In multivariable analysis, adjuvant treatment was associated with improved survival (HR 0.76; $95 \%$ CI $0.62-0.92, p=0.005)$. Patients receiving adjuvant therapy were more likely to be younger, had fewer comorbidities, had greater posttreatment tumor burden, tumor grade, or received induction chemotherapy without radiation. Patients receiving adjuvant therapy also were less likely to have had postoperative complications, such as infection.

Despite these retrospective studies suggesting a clinical benefit with adjuvant chemotherapy after induction therapy followed by esophagectomy, the routine use of adjuvant chemotherapy has not been recommended. High-level, prospective data are lacking to support the use of adjuvant chemotherapy. Questions remain regarding the choice of adjuvant chemotherapeutic agents based on the neoadjuvant treatment. It is unclear whether the same chemotherapy should be continued in the adjuvant setting despite lack of histologic response or if different chemotherapy agents should be considered that risks additional morbidity and mortality without demonstrated clinical efficacy. ${ }^{9,10}$ Most importantly, adjuvant chemotherapy often is difficult to administer safely in these patients who have already received extensive treatment, including toxic chemoradiation followed by a challenging operation that may require months of recovery time. The toxicities inherent to chemotherapy often are magnified post resection and may place patients at high risk of lifethreatening complications.

In this context, Kelly et al. ${ }^{11}$ for the CheckMate 577 Investigators reported the interim findings of a seminal study that demonstrates the impactful clinical benefit of adjuvant immunotherapy following trimodality therapy for esophageal or gastroesophageal junction carcinoma. Patients with esophageal squamous cell carcinoma or adenocarcinoma, stage 2 or 3 (AJCC/UICC Cancer Staging Manual, 7th edition), who had completed concurrent preoperative chemoradiation followed by resection and did not achieve a pathologic complete response were randomized to either placebo or the PD1-inhibitor, nivolumab, for 1 year beginning 4 to 16 weeks after surgery. It is important to note that subject selection and randomization occurred only after they had recovered from resection, thereby excluding patients who could not or did not proceed to surgery and those treated with chemotherapy and definitive dose radiation therapy, without resection. 
Of 1,085 patients who were evaluated between 2016 and 2019, 794 were eligible and assigned in a 2:1 ratio to receive either nivolumab (532 patients) or placebo (262 patients). The median follow-up time was 24.4 (range, 6.2-44.9) months. The primary endpoint-disease-free survival-was significantly longer for patients receiving nivolumab, 22.4 months (95\% CI 16.6-34.0), versus placebo, 11.0 months (95\% CI 8.3-14.3) (HR 0.69; $96.4 \%$ CI $0.56-0.86 ; p<0.001)$. For this interim report, the secondary outcome of overall survival was not reported.

Patients with either major histologic subtype of esophageal cancer appeared to derive benefit in post-hoc analysis. Patients with adenocarcinoma demonstrated a disease-free survival of 19.4 months (95\% CI 15.9-29.4) with adjuvant nivolumab versus 11.1 months (95\% CI 8.316.8) with placebo (HR $0.75 ; 95 \%$ CI 0.59-0.96). Patients with squamous cell carcinoma had a disease-free survival of 29.7 months (95\% CI 14.4 to $\mathrm{NE}$ ) with nivolumab compared with 11.0 months (95\% CI 7.6-17.8) with placebo (HR 0.61; 95\% CI 0.42-0.88). Median disease-free survival for patients with residual nodal disease who received nivolumab was 14.8 months versus 7.6 months with placebo (unstratified HR 0.67 ; 95\% CI 0.53-0.86)). Distant and locoregional recurrence occurred less frequently in the nivolumab-treated cohort; median distant metastasis-free survival was 28.3 months (95\% CI 21.3 to $\mathrm{NE})$ with nivolumab versus 17.6 months (95\% CI 12.5-25.4) with placebo (HR 0.74; 95\% CI 0.60-0.92).

Adverse events related to nivolumab included fatigue, diarrhea, pruritus, and rash, occurring in $8 \%$ of patients, whereas in the placebo group, diarrhea and fatigue were the most common adverse events, occurring in $3 \%$ of patients. Health-related quality of life was maintained and did not differ significantly between the nivolumab and placebotreated groups. Kelly et al. recently provided an update at the 2021 American Society of Clinical Oncology Annual Meeting confirming that this adjuvant immunotherapy was shown to be safe and maintained quality of life compared with the placebo-control treatment group. ${ }^{12}$

These findings establish a new standard of care for patients who have completed trimodality therapy, for whom residual carcinoma is identified on pathologic review postresection. It is important to note that this practice-changing trial is applicable to a fairly narrow patient population who received trimodality therapy without a pathologic complete response. Patients who have a pathologic complete response to trimodality therapy have improved survival compared with those who have partial or no response but are still at high risk for the development of recurrent incurable malignancy. In addition, questions regarding the benefits of immunotherapy in other treatment strategies for locoregionally advanced esophageal cancer have yet to be answered and are the focus of ongoing trials.
In the KEYNOTE-585 (NCT03221426) phase III, multicenter, randomized, double-blind study, subjects with resectable gastric or esophagogastric junction cancer will be treated with perioperative chemotherapy (FLOT: 5-fluorouracil, leucovorin, oxaliplatin, and docetaxel) or cisplatin/fluoropyrimidine-based therapy and are randomized to adjuvant pembrolizumab versus placebo. ${ }^{13}$ Primary endpoints include overall survival, event-free survival, and pathologic complete response. In a separate, ongoing study-KEYNOTE-975 (NCT04210115) study, patients with esophageal cancer (squamous or adenocarcinoma histology) or esophagogastric junction carcinoma, who are deemed unresectable or who decline surgery, are treated with definitive chemoradiation, including FP or FOLFOX (5-fluorouracil, leucovorin, and oxaliplatin) followed by consolidation pembrolizumab vs. placebo. ${ }^{14}$ Primary endpoints for this study include overall survival and event-free survival.

While these ongoing studies may provide clarity for broadening the use of immune checkpoint inhibitor therapy in the treatment of locally advanced esophageal cancer, the findings from CheckMate 577, by Kelly et al., demonstrate that the addition of adjuvant immune checkpoint inhibitor therapy with nivolumab confers a significant reduction in cancer recurrence and provide hope for those patients who do not achieve a complete pathologic response to neoadjuvant chemoradiation postresection. This study may provide a unique paradigm for other solid tumors treated with neoadjuvant therapy followed by resection and have a less than favorable response to the standard multimodality treatment.

\section{REFERENCES}

1. Siegel RL, Miller KD, Fuchs HE, Jemal A. Cancer statistics, 2021. CA Cancer Journal Clin. 2021;71(1):7-33.

2. Shapiro J, van Lanschot JJB, Hulshof MCCM, et al. Neoadjuvant chemoradiotherapy plus surgery versus surgery alone for oesophageal or junctional cancer (CROSS): long-term results of a randomised controlled trial. Lancet Oncol. 2015;16(9):1090-8.

3. Al-Batran S-E, Homann N, Pauligk C, Goetze TO, Meiler J, Kasper S, et al. Perioperative chemotherapy with fluorouracil plus leucovorin, oxaliplatin, and docetaxel versus fluorouracil or capecitabine plus cisplatin and epirubicin for locally advanced, resectable gastric or gastro-oesophageal junction adenocarcinoma (FLOT4): a randomised, phase $2 / 3$ trial. Lancet. 2019;393(10184):1948-57.

4. Cunningham D, Allum WH, Stenning SP, Thompson JN, Van de Velde CJH, Nicolson M, et al. Perioperative chemotherapy versus surgery alone for resectable gastroesophageal cancer. $N$ Engl $J$ Med. 2006;355(1):11-20.

5. Ychou M, Boige V, Pignon J-P, Conroy T, Bouché O, Lebreton $\mathrm{G}$, et al. Perioperative chemotherapy compared with surgery alone for resectable gastroesophageal adenocarcinoma: an FNCLCC and FFCD multicenter phase III trial. J Clin Oncol. 2011;29(13):1715-21. 
6. van Hagen P, Hulshof MCCM, van Lanschot JJB, Steyerberg EW, Henegouwen MI van B, Wijnhoven BPL, et al. Preoperative chemoradiotherapy for esophageal or junctional cancer. $N$ Engl $J$ Med. 2012;366(22):2074-84.

7. Samson P, Puri V, Lockhart AC, Robinson C, Broderick S, Patterson GA, et al. Adjuvant chemotherapy for patients with pathologic node-positive esophageal cancer after induction chemotherapy is associated with improved survival. $J$ Thorac Cardiovasc Surg. 2018;156(4):1725-35.

8. Semenkovich TR, Subramanian M, Yan Y, Hofstetter WL, Correa $\mathrm{AM}$, Cassivi SD, et al. Adjuvant therapy for node positive esophageal cancer after induction and surgery: a multisite study. Ann Thorac Surg. 2019;108(3):828-36.

9. Stiles BM, Christos P, Port JL, Lee PC, Paul S, Saunders J, et al. Predictors of survival in patients with persistent nodal metastases after preoperative chemotherapy for esophageal cancer. $J$ Thorac Cardiovasc Surg. 2010;139(2):387-94.

10. Burt BM, Groth SS, Sada YH, Farjah F, Cornwell L, Sugarbaker DJ, et al. Utility of adjuvant chemotherapy after neoadjuvant chemoradiation and esophagectomy for esophageal cancer. Ann Surg [Internet]. 2017;266(2). Available at: https://journals.lww.c om/annalsofsurgery/Fulltext/2017/08000/Utility_of_Adjuvant_C hemotherapy_After_Neoadjuvant.16.aspx
11. Kelly RJ, Ajani JA, Kuzdzal J, Zander T, Van Cutsem E, Piessen $\mathrm{G}$, et al. Adjuvant nivolumab in resected esophageal or gastroesophageal junction cancer. $N$ Engl $J$ Med. 2021;384(13):1191-203.

12. Kelly RJ, Ajani JA, Jaroslaw K, et al. Adjuvant nivolumab (NIVO) in resected esophageal or gastroesophageal junction cancer (EC/GEJC) following neoadjuvant chemoradiotherapy (CRT): Expanded efficacy and safety analyses from CheckMate 577. J Clin Oncol. 2021;39(suppl 15).

13. Bang Y-J, Van Cutsem E, Fuchs CS, Ohtsu A, Tabernero J, Ilson $\mathrm{DH}$, et al. KEYNOTE-585: Phase III study of perioperative chemotherapy with or without pembrolizumab for gastric cancer. Future Oncol. 2019;15(9):943-52.

14. Shah MA, Bennouna J, Doi T, Shen L, Kato K, Adenis A, et al. KEYNOTE-975 study design: a Phase III study of definitive chemoradiotherapy plus pembrolizumab in patients with esophageal carcinoma. Future Oncol. 2021;17(10):1143-53.

Publisher's Note Springer Nature remains neutral with regard to jurisdictional claims in published maps and institutional affiliations. 